\title{
The Prevalence of Malaria in Tselemti Wereda, North Ethiopia: A Retrospective Study
}

\author{
Meresa Shiferaw ${ }^{1}$, Megbaru Alemu ${ }^{2 *}$, Kiros Tedla ${ }^{3}$, Desalegn Tadesse ${ }^{3}$, Sena \\ Bayissa $^{3}$, Gessessew Bugssa ${ }^{3}$
}

\footnotetext{
OPEN ACCESS

Citation: Abdissa Biruksew, Ahmed Zeynudin, Yonas Alemu, Lemu Golassa, Moti Yohannes, Asfaw Debella, Geme Urge, Bart De Spiegeleer, Sultan Suleman. Ethiop J Health Sci.2018;28 (5):539.

doi:http://dx.doi.org/10.4314/ejhs.v28i5.4 Received: January 11, 2018

Accepted: January 11, 2018

Published: September 1, 2018

Copyright: (C) 2018 Meresa Shiferaw et al. This is an open access article distributed under the terms of the Creative Commons Attribution License, which permits unrestricted use, distribution, and reproduction in any medium, provided the original author and source are credited.

Funding: Nil

Competing Interests: The authors declare that this manuscript was approved by all authors in its form and that no competing interest exists.

Affiliation and Correspondence:

${ }^{1}$ Maytsebri Health Center, Tigray

regional state, North Ethiopia

${ }^{2}$ Department of Microbiology,

Immunology and Parasitology, Bahir

Dar University, Ethiopia ${ }^{5}$ Aklilu

Lemma Institute of Pathobiology,

Addis Ababa University

${ }^{3}$ Department of Medical Parasitology

and Vector Biology, Mekelle

University, Ethiopia

*Email: mgbeyney@gmail.com
}

\section{ABSTRACT}

BACKGROUND: A significant segment of the world's population is at risk of contracting malaria infection at any one time. In Ethiopia, sustained control efforts have been made in the past decade to fight malaria. Yet, it remains as the major cause of morbidity, mortality and socioeconomic problems in the country. The intensified control of malaria can further be augmented by analyzing health facility based malaria data. Hence, the aim of this study was to determine the magnitude of malaria infection in Northwest Ethiopia.

METHODS: $A$ retrospective record review was conducted in Northwest Ethiopia from February-April 2016. All blood film results reported between January 2013 and December 2015 in the seven health centers were extracted and analyzed.

RESULTS: A total of 41,773 patients with chief malaria complaint were screened for malaria in the three years period. The overall prevalence of microscopically confirmed malaria was $28.1 \%$. Males (29.5\%) were more affected by malaria than females (26.5\%). Malaria was also higher in the age group $>15$ years (32.6\%) followed by 5-15 years (29.3\%) and under-five children (20.5\%). Plasmodium falciparum, Plasmodium vivax and mixed infections accounted for $58.2 \%, 35.5 \%$ and $6.3 \%$, respectively. The highest prevalence of confirmed malaria cases was observed during spring (35.6\%) and summer (25.1\%). Higher prevalence of slide positive malaria was recorded in Dima (46.1\%), Cherecher (45.3\%) and Fyel wuha (35.3\%) health centers.

CONCLUSION: Malaria specific outpatient cases were high in the study area. Both plasmodia species were of public health significance in the area with predominance of Plasmodium falciparum. KEYWORDS: Prevalence, Tselemti, Wereda, Malaria, Ethiopia 


\section{INTRODUCTION}

Malaria has so far been a life-threatening parasitic disease transmitted by the bite of female anopheles mosquitoes. About half of the world population (3.3 billion) is at risk of malaria infection, and around 250 million cases occur annually, leading to approximately 1 million deaths each year (1).The disease is the leading cause of death in children under the age of five and pregnant women in developing countries (2). The vast majority of cases reside in the African continent followed by southeast Asia and Eastern Mediterranean regions (3). The disease remains one of the most important causes of human morbidity and mortality with enormous medical and economic impact in the world (4).

In sub-Saharan Africa, the pattern of malaria transmission varies markedly from region to region, depending on climate and biogeography and broad ecological categories (5). In Ethiopia, Plasmodium falciparum and Plasmodium vivax are the two predominant plasmodium species distributed all over the country, accounting for $60 \%$ and $40 \%$ of malaria cases, respectively. The main malaria control strategies in Ethiopia include, but not limited to, early diagnosis and prompt treatment, vector control, epidemic management and personal protection through the use of insecticide treated nets (6). Malaria transmission in Tigray is very seasonal, and most of the areas in northwestern and western zones are favorable to mosquito breeding and occur mainly below 2,200 meter above sea level (7). It is also disseminated to the previous malaria free areas of the region putting the non-immuned population at risk of contracting malaria in the present time (8).

Incredible progress has been attained recently in Ethiopia in fight against malaria. This was possible partly due to engagement of the health extension workers (HEWs) and the health development army (HAD) in the preventive and case management interventions at the household level (9). However, there is insufficient malaria data from many sub-Saharan African countries where Ethiopia is no exception (10). Health facility based prevalence studies can be used to enhance understanding of the status of malaria in an area/region. The findings can also be used to influence decision makings of malaria prevention strategies. Moreover, the status of malaria is not yet determined in the present study area. Hence, the aim of this study was to determine the prevalence of malaria at 7 Health Centers in Tselemti wereda, Northwest Ethiopia.

\section{METHODS AND MATERIALS}

Study area and period: This cross-sectional retrospective study was conducted in Tselemti Wereda, Northwest Ethiopia, from February-April 2016. It is located 1200 kilometers from Addis Ababa at an altitude of 1400 meters above sea level with $14^{0} 40^{\prime} 0.00^{\prime}$ north latitudes and $38^{\circ}$ $10^{\prime} 0,00^{\prime}$ east longitudes. The area is mainly lowland with a temperature range of $30-35^{\circ} \mathrm{C}$, and mean annual rainfall of $350-750 \mathrm{~mm}$. There were seven health centers in the study area, namely; Maytsebre, Tsadakerni, Mayani, Sekota, Dima, Chachere and Fyel Wuha. Each health center is believed to serve an average of 22,000 people in the catchment areas.

Sample size: All blood film results reported and registered in the laboratory logbook in the period of 2013-2015 were reviewed and included in this retrospective study.

Data collection: Data extraction sheets were prepared to collect sociodemographic and laboratory data from patients' registration book.

Malaria diagnosis: In all health centers, blood smear examination was performed via Giemsa stained blood films prepared from capillary blood used as the gold standard in microscopic investigation of malaria. Both thin and thick films were prepared in the health centers and standard operating procedures (SOPs) were strictly followed in preparation and examination of blood films. Both thick and thin blood films were prepared. In the thick film preparation, three drops of blood were distributed over an area of $1 \mathrm{~cm} 2$. Thin smear on the other hand was prepared by evenly distributing a drop of blood on a grease free microscopic slide. Slides were labeled, dried, fixed with methanol alcohol (thin smear only), and were stained (using 3\% Giemsa stain solution for 30 minutes). The stained blood film was washed

DOI: http://dx.doi.org/10.4314/ejhs.v28i5.4 
with distilled water and it was air dried. Detection and species identification of the plasmodium parasites were made via thick and thin blood films, respectively.

Data analysis: Data was entered and analyzed using SPSS 20 software package (IBM Comp. released 2011.IBM SPSS statistics for windows, version 20Armonk, NY: IBM comp.). Crosstabs were used to get the frequency distribution of variables. Malaria infection was sorted in terms of health centers, Plasmodium species, sex and age groups. Finally, the data was described and presented using tables.

Ethics approval and consent to participate: Ethical approval was obtained from Mekelle University; College of Health Institutional Review Board (IRB). Permission letter was provided by Tigray Regional Health Bureau to utilize the data. Confidentially was maintained as no details related to patients' identity were used.

\section{RESULTS}

A total of 41,773 presumptive malaria patients gave blood for microscopic investigation of malaria parasites in the three years period. Males accounted for $54.1 \%$ of the participants. Most of the study participants were above 15 years of age $(40.5 \%)$, followed by $5-15$ years $(30.6 \%)$ and under-five patients $(28.9 \%)$ (Table 1$)$.

Out of the total malaria complaints, 11,745 were confirmed malaria infections, making the overall slide positivity rate of malaria $28.1 \%$. The two species of plasmodium were responsible for malaria morbidity in the area. Plasmodium falciparum infection accounted for $58.2 \%$ while $35.5 \%$ of the infections were due to $P$. vivax. Mixed infection by both P.falciparum and $P$. vivax on the other hand accounted for $6.3 \%$. The overall prevalence of malaria was higher in males $(29.5 \%)$ than in females $(26.5 \%)$. The majority of the reported cases were in the age group of $>15$ years $(32.6 \%)$ followed by $5-15$ years $(29.3 \%)$ and under-5 children $(20.5 \%)$ (Table 1$)$.

Higher rates of confirmed malaria cases were reported during spring (Sep-Nov) (35.6\%) followed by summer (June-August) (25.1\%), winter (December-February) (24\%) and autumn (March-May) (23.4\%) (Figure 1).

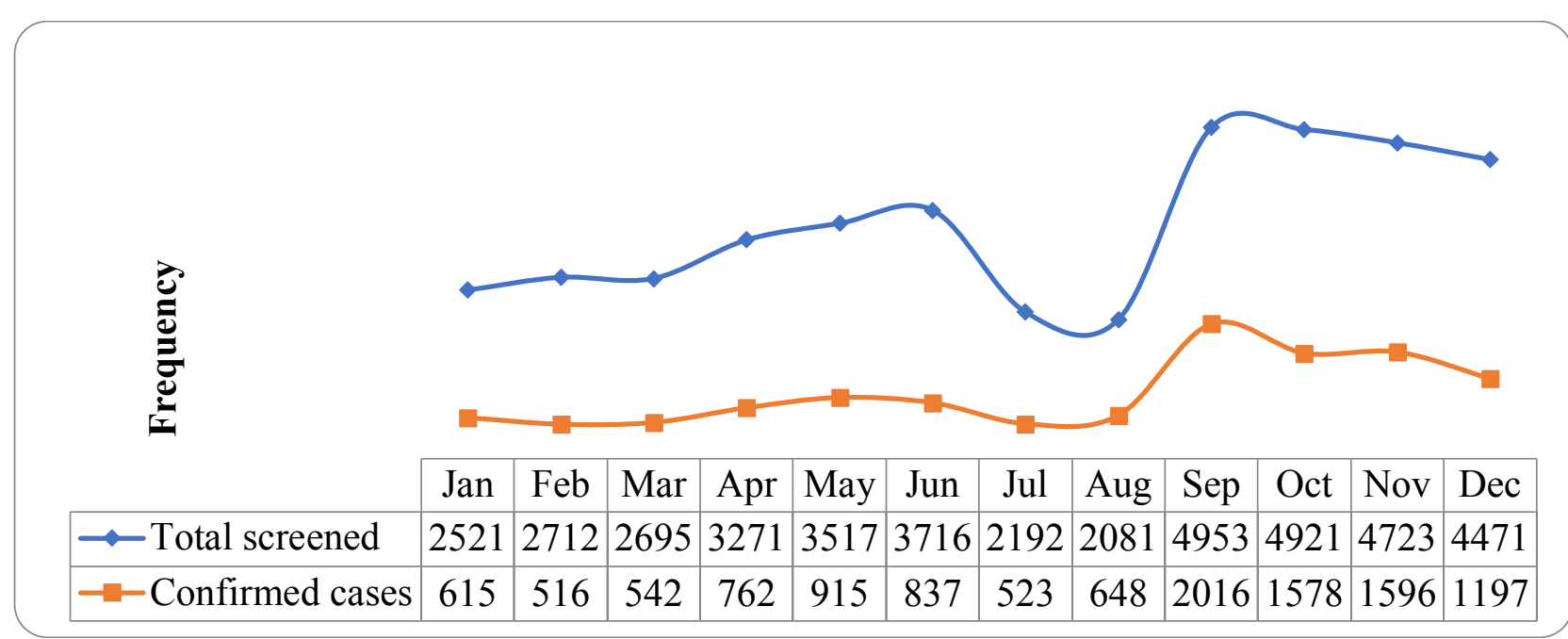

Figure 1: Annual distribution of malaria in Tselemti wereda, North Ethiopia, 2013-2015

The prevalence of confirmed malaria cases was higher in Dima health center (46.1\%) followed by Cherecher (45.3\%), Fyel Wuha (35.3\%) and Sekota $(28.7 \%)$ health centers (Table 2). The highest prevalence of $P$. falciparum (30.6\%) was reported in Fyel Wuha Health Center. On the other hand, higher rate of $P$. vivax infection was recorded in Cherecher Health Center. Mixed infections were higher in Dima Health Center $(3.7 \%)$. The overall prevalence of malaria specific

DOI: http://dx.doi.org/10.4314/ejhs.v28i5.4 
outpatient cases showed no remarkable decrease over the years; $32.3 \%, 28.1 \%$ and $25 \%$ at 2013 , 2014 and 2015, respectively. However, malaria prevalence increased in some health facilities;

Table 1: Socio-demographic characteristics of the study participants and distribution of malaria in Tselemti

Wereda ,North Ethiopia, 2013-2015.

\begin{tabular}{|c|c|c|c|}
\hline Variables & No screened & No positive & Percentage \\
\hline \multicolumn{4}{|l|}{ Gender } \\
\hline Males & 22605 & 6662 & 29.5 \\
\hline Females & 19168 & 5083 & 26.5 \\
\hline \multicolumn{4}{|l|}{ Age } \\
\hline$<5$ & 12096 & 2485 & 20.5 \\
\hline $5-15$ & 12764 & 3739 & 29.3 \\
\hline $15+$ & 16913 & 5521 & 32.6 \\
\hline \multicolumn{4}{|l|}{ Month } \\
\hline January & 2521 & 615 & 24.4 \\
\hline February & 2712 & 516 & 19.0 \\
\hline March & 2695 & 542 & 20.1 \\
\hline April & 3271 & 762 & 23.3 \\
\hline May & 3517 & 915 & 26.0 \\
\hline June & 3716 & 837 & 22.5 \\
\hline July & 2192 & 523 & 23.9 \\
\hline August & 2081 & 648 & 31.1 \\
\hline September & 4953 & 2016 & 40.7 \\
\hline October & 4921 & 1578 & 32.1 \\
\hline November & 4723 & 1596 & 33.8 \\
\hline December & 4471 & 1197 & 26.8 \\
\hline \multicolumn{4}{|l|}{ Year } \\
\hline 2013 & 11301 & 3649 & 8.7 \\
\hline 2014 & 15639 & 4392 & 10.5 \\
\hline 2015 & 14833 & 3704 & 8.9 \\
\hline \multicolumn{4}{|l|}{ Plasmodium species } \\
\hline P. falciparum & 41773 & 6835 & 58.2 \\
\hline P. vivax & 41773 & 4165 & 35.5 \\
\hline Mixed $(P f+P v)$ infection & 41773 & 745 & 6.3 \\
\hline \multicolumn{4}{|l|}{ Health facility } \\
\hline Maytsebri & 22849 & 4823 & 21.1 \\
\hline Dima & 6360 & 2930 & 46.1 \\
\hline Sekota & 2876 & 824 & 28.7 \\
\hline Mayayni & 3888 & 1001 & 25.7 \\
\hline Chachere & 3319 & 1504 & 45.3 \\
\hline Fyelwuha & 170 & 60 & 35.3 \\
\hline Tsaedakerni & 2311 & 603 & 26.1 \\
\hline
\end{tabular}

namely, Mayayni $(19.4 \%$ in 2013 and $26.6 \%$ in 2015) and Fyel Wuha (27.3\% in 2013 and $42.9 \%$ in 2015) (Table 1).

DOI: http://dx.doi.org/10.4314/ejhs.v28i5.4 
The Prevalence of Malaria in Tselemti...

Meresa S. et al.

Table 2: Slide positive rate of malaria at the seven health centers in Tselemti wereda North Ethiopia, 20132015.

\begin{tabular}{|c|c|c|c|c|c|}
\hline Health facility & Years & $\operatorname{Pfn}(\%)$ & $P v \mathrm{n}(\%)$ & Mixed n (\%) & Total n (\%) \\
\hline \multirow[t]{4}{*}{ Maytsebri } & $2013(n=7901)$ & $1229(15.6)$ & $802(10.2)$ & $94(1.2)$ & $2125(26.9)$ \\
\hline & $2014(\mathrm{n}=8302)$ & $1039(12.5)$ & $655(7.9)$ & $143(1.7)$ & $1837(22.1)$ \\
\hline & $2015(\mathrm{n}=6646)$ & $456(6.9)$ & $316(4.8)$ & $89(1.3)$ & 861(13.0) \\
\hline & Total $(n=22849)$ & 2724 (11.9) & $1773(7.8)$ & $326(1.4)$ & $4823(21.1)$ \\
\hline \multirow[t]{4}{*}{ Dima } & $2013(\mathrm{n}=2451)$ & $722(29.5)$ & $470(19.2)$ & $91(3.7)$ & $1283(52.3)$ \\
\hline & $2014(\mathrm{n}=2092)$ & $466(22.3)$ & $320(15.3)$ & $76(3.6)$ & $862(41.2)$ \\
\hline & $2015(\mathrm{n}=1817)$ & $423(23.3)$ & $295(16.2)$ & $67(3.7)$ & $785(43.2)$ \\
\hline & Total $(n=6360)$ & $1611(25.3)$ & $1085(17.1)$ & $234(3.7)$ & $2930(46.1)$ \\
\hline \multirow[t]{4}{*}{ Sekota } & $2013(n=445)$ & $115(25.8)$ & $25(5.6)$ & $3(0.7)$ & $143(32.1)$ \\
\hline & $2014(\mathrm{n}=1232)$ & $223(18.1)$ & $117(9.5)$ & $6(0.5)$ & $346(28.1)$ \\
\hline & $2015(\mathrm{n}=1199)$ & 201(16.8) & $122(10.2)$ & $12(1.0)$ & $335(27.9)$ \\
\hline & Total $(n=2876)$ & $539(18.7)$ & $264(9.2)$ & $21(0.7)$ & $824(28.7)$ \\
\hline \multirow[t]{4}{*}{ Mayayni } & $2013(\mathrm{n}=504)$ & $61(12.1)$ & $37(7.3)$ & - & $98(19.4)$ \\
\hline & $2014(\mathrm{n}=1461)$ & $322(22.0)$ & $52(3.6)$ & $17(1.2)$ & $391(26.8)$ \\
\hline & $2015(n=1923)$ & $282(14.7)$ & $198(10.3)$ & $32(1.7)$ & $512(26.6)$ \\
\hline & Total $(n=3888)$ & $665(17.1)$ & $287(7.4)$ & $49(1.3)$ & $1001(25.7)$ \\
\hline \multirow[t]{4}{*}{ Chachere } & 2013 & - & - & - & - \\
\hline & $2014(n=1607)$ & $408(25.4)$ & $279(17.4)$ & $37(2.3)$ & $724(45.1)$ \\
\hline & $2015(\mathrm{n}=1712)$ & $430(25.1)$ & 294(17.2) & $56(3.3)$ & $780(45.6)$ \\
\hline & Total $(n=3319)$ & $838(25.2)$ & $573(17.3)$ & $93(2.8)$ & $1504(45.3)$ \\
\hline \multirow[t]{4}{*}{ Fyel wuha } & 2013 & - & - & - & - \\
\hline & $2014(\mathrm{n}=55)$ & $12(21.8)$ & $3(5.5)$ & - & $15(27.3)$ \\
\hline & $2015(n=105)$ & $37(35.2)$ & $4(3.8)$ & $4(3.8)$ & $45(42.9)$ \\
\hline & Total $(n=170)$ & $49(28.8)$ & $7(4.1)$ & $4(2.4)$ & $60(35.3)$ \\
\hline \multirow[t]{4}{*}{ Tsaeda kerni } & 2013 & - & - & - & - \\
\hline & $2014(\mathrm{n}=890)$ & 161(18.1) & $49(5.5)$ & $7(0.8)$ & $217(24.4)$ \\
\hline & $2015(n=1421)$ & $248(17.5)$ & $127(8.9)$ & $11(0.8)$ & $386(27.2)$ \\
\hline & Total $(n=2311)$ & $409(17.7)$ & $176(7.6)$ & $18(0.8)$ & $603(26.1)$ \\
\hline Total & $(\mathrm{N}=41773)$ & $6835(16.4)$ & $4165(10.0)$ & $745(1.8)$ & $11745(28.1)$ \\
\hline
\end{tabular}

$\mathrm{Pf}=$ Plasmodium falciparum; $\mathrm{Pv}=$ Plasmodium viva

DOI: http://dx.doi.org/10.4314/ejhs.v28i5.4 


\section{DISCUSSION}

Malaria remains one of the most important causes of morbidity and mortality with enormous medical, economic and emotional impact in the world. In Ethiopia, it is a major public health significance, and it has been consistently reported as the first leading cause of outpatient visits, hospitalization and death in health facilities across the country (11). Our study revealed that the overall slide positive rate of malaria was $(28.1 \%)$. This result was higher than similar studies done in Northwest Ethiopia (12 and Southern Ethiopia (13). However, it was lower than the findings reported from Kola Diba, Ethioia (14). This might possibly be due to differences among areas in suitability for breeding of Anopheles vector which are mainly altitude differences and climatological variations.

Both $P$. falciparum and $P$. vivax were identified in the area and $P$. falciparum $(58.2 \%)$ was the predominant species across all health centers followed by $P$. vivax $(35.5 \%)$ and mixed infections $(6.3 \%)$. This goes in line with other previous studies (11,15-17). However, P. vivax was found to be predominant species in several other studies $(18,19)$.

According to our study, males were more affected by malaria than females. This was in agreement with other results in Ethiopia and elsewhere in the world. The higher prevalence rate in males might be due to the fact that males are usually engaged in outdoor activities at dusks and dawns which coincide with the peak biting hours of the exophagic Anopheles mosquito vectors $(11,20)$.

This study also showed that malaria was reported in all age groups in the study area and the age groups $>15$ years were more affected. It was in line with the findings from Kola Diba (14), Kersa (11) and Wolaita Zone, Ethiopia (20). However, contradictory results were reported in Arsi Negelle and Metema, Ethiopia $(12,13)$. This could be because people in this age group are involved in agricultural activities that may require spending outdoors during peak biting activities of mosquitoes to sustain livelihood of the family. Besides, they prefer to offer mosquito nets for the sake of the wellbeing of their family members mainly for children and pregnant women (11).

According to the national Plasmodium species distribution in Ethiopia, $P$. falciparum and $P$. vivax are the two predominant species occurring in the country with $60 \%: 40 \%$ composition, respectively (21). In our study, the predominantly reported species was $P$. falciparum with $52.2 \%$ of the overall occurrence followed by $P$. vivax $(35.5 \%)$ and mixed infection of both species $(6.3 \%)$. This is in agreement with findings from various studies (11-13,20). Another study conducted in Oromia by Deresssa et al (22) found that the species composition of plasmodia was; $P$. falciparum $(51.5 \%)$ and $P$. vivax $(32.3 \%)$. On the other hand, Yewhalaw et al. (23) reported predominance of $P$. vivax over $P$. falciparum. Woyessa et al. (19) reported the predominance of $P$. falciparum during October while $P$. vivax tends to dominate during November.

In this study, $6.3 \%$ of mixed malarial infection of both $P$. falciparum and $P$. vivax was found. This was higher than previous studies (1113) and indicates co-existence of the two species which jeopardize the livelihood of the community in the area.

In the current study area, malaria was reported in all the 12 months of the year. However, the highest prevalence of confirmed malaria cases was observed during spring (September-November) followed by summer (June-August) and autumn (December-February). This goes in line with other studies in Ethiopia $(12,13,25)$. This could be attributed to the high temperature and rainfall which are the potential predicators of malaria during these seasons.

The prevalence of malaria as evident with slide positivity rate of $28.1 \%$ was high in the study area. It was higher in males and in the age group of $>15$ years. $P$. falciparum was the predominant species. Malaria specific outpatient cases showed no remarkable decline in Tselemti wereda. Both species of plasmodium are public health problems in the area with predominance of the deadly species, P. falciparum. Due to its retrospective nature, the study lacks detailed clinical picture of 
patients which might have a determinant factor to show the complete picture of the study subjects.

\section{ACKNOWLEDGMENT}

We are thankful to the laboratory personnel and clinicians at the health centers.

\section{REFERENCES}

1. WHO (world health organization); health impact assessment, $1^{\text {st }}$ inter ministerial conference in Africa, Geneva Switzerland; 2008.

2. World Health Organization. Global technical strategy for malaria 2016-2030; 20 Avenue Appia, 1211 Geneva 27, Switzerland. Available at: <www.who.int/about/licensing/ copyright form/en/index.html $>$ Accessed on 7 May 2017.

3. World Health Organization: World malaria report. Geneva, Switzerland; 2011.

4. Snow RW, Guerra CA, Noor AM, Myint HY et al. The global distribution of clinical episodes of Plasmodium falciparum malaria. Nature 2005;434: 214-7.

5. World Health Organization,World Malaria Report, Key points, Geneva, Switzerland, 2014.

6. Ministry of Health: Guidelines for malaria vector control in Ethiopia. Malaria control profile 2004.

7. THB Tigray health profile 2005/2006, Mekelle HMIS unit Tigray health bureau; 2006.

8. Hadgu Gerensea and Hafte Teklay. Pattern and Trend of Malaria Morbidity and Mortality in Tigray Region, Ethiopia from 2011/12-2014/15. J Bioanal Biomed. 2017;9(2).

9. The Ethiopian Health and Nutrition Research Institute and partners. Ethiopia National Malaria Indicator Survey; Addis Ababa, Ethiopia, 2011.

10. WHO Global malaria programme. World malaria report 2014; Gneva Switzerland.

11. Kaliyaperumal Karunamoorthi, Mammo Bekele. Prevalence of malaria from peripheral blood smears examination: A 1- year retrospective study from the Serbo Health Center, Kersa Woreda, Ethiopia. J Infect Public Health 2009;2:171-176.

12. Getachew F, Abiyu W, Alemtegna G, Ali A et al. Prevalence of malaria from blood smears examination: A seven- year retrospective study from Metema Hospital, Northwest Ethiopia. Malar Res Treat. 2013.

13. Mengistu Hailemariam and Solomon Gebre. Trend analysis of malaria prevalence in Arsi Negelle health center, Southern Ethiopia. $J$ Infect Dis Immun. 2015;7(1).

14. Alemu A, Muluye D, Mihret M, Adugna M et al. Ten year trend analysis of malaria prevalence in Kola Diba, North Gonder, Northwest Ethiopia. Parasit Vectors 2012;5:173.

15. Federal Republic of Ethiopia Ministry of Health, National Guide Lines, Federal Republic of Ethiopia ministry of health, Addis Abeba, Ethiopia, 3rd edition, 2012.

16. Asnakew K, Sucharita G, Afework T, Dereje $\mathrm{D}$ et al. Spatial analysis of malaria incidence at the village level in areas with unstable transmission in Ethiopia. Int $J$ Health Geogr. 2009;8:.5-16.

17. Manuel Ramos J, Reyes F, Tesfamariam A. Change in epidemiology of malaria infections in a rural area in Ethiopia. $J$ Travel Med .2005;12(3):155-156.

18. Solomon T, Yeshambel B, Takele T, Girmay $\mathrm{M}$ et al. Malaria pattern observed in the highland fringe of Butajira, Southern Ethiopia: a ten-year retrospective analysis from parasitological and metrological data. MWJ 2012;3(5).

19. Woyessa A, Teshome Gebre-Michael, and Ahmed Ali. An indigenous malaria transmission in the outskirts of Addis Ababa, Akaki Town and its environs. Ethiop $J$ Health Dev. 2004;18:2-7.

20. Deresse Legesse, Yusuf Haji, and Solomon Abreha. Trend Analysis of Malaria Occurrence in Wolaita Zone, Southern Ethiopia: Retrospective Cross-Sectional Study. Malar Res Treat. 2015.

21. National five-year strategic plan for malaria control in Ethiopia: 2001-2005. Addis Ababa, 
Ethiopia: Malaria and Other Vector borne Diseases Prevention and Control Unit, Disease Prevention and Control Department; 2001.

22. Wakgari Deressa, Shelleme Chibsa, Dereje Olana. The distribution and magnitude of malaria in Oromia, Ethiopia. Ethiop J Health Dev. 2004;18(3):164-70.

23. Yewhalaw D, Legesse W, Van Bortel W, Gebre-Selassie $\mathrm{S}$ et al. Malaria and water resource development: the case of GilgelGibe hydroelectric dam in Ethiopia. Malar $J$. 2009;8:21.

24. Mulat Yimer, Tadesse Hailu, Wondemagegn Mulu, Bayeh Abera et al. A 5 year trend analysis of malaria prevalence within the catchment areas of Felegehiwot referral Hospital, Bahir Dar city, northwest Ethiopia: a retrospective study. BMC Res Notes 2017;10:239. 